Research Paper

\title{
The Expression of Toll-like Receptor 8 and Its Relationship with VEGF and Bcl-2 in Cervical Cancer
}

\author{
Yun Zhang1, Heng Yang1, Prince Amoah Barnie ${ }^{1}$, Peifang Yang ${ }^{2}$, Zhaoliang Su ${ }^{1}$, Jianguo Chen², Zhijun \\ $\mathrm{Jiao}^{2}$, Liwei $\mathrm{Lu}^{3}$, Shengjun Wang ${ }^{\bowtie}$, Huaxi Xu${ }^{1^{\bowtie}}$ \\ 1. Department of Immunology, School of Medical Science and Laboratory Medicine, Jiangsu University, Zhenjiang 212013, PR China \\ 2. Department of gynaecology and obstetrics, Affiliated Hospital of Jiangsu University, Zhenjiang 212001, PR China \\ 3. Department of Pathology and Centre of Infection and Immunology, The University of Hong Kong, Hong Kong 999077, PR China
}

$\triangle$ Corresponding authors: Huaxi Xu, Shengjun Wang; Dept of Immunology, School of Medical Science and Laboratory Medicine, Jiangsu University, Xuefu road 301, Zhenjiang, PR China. Phone: +86 511 88791048; Fax: +86 511 88791739; Email: xuhx@ujs.edu.cn

(c) Ivyspring International Publisher. This is an open-access article distributed under the terms of the Creative Commons License (http://creativecommons.org/ licenses/by-nc-nd/3.0/). Reproduction is permitted for personal, noncommercial use, provided that the article is in whole, unmodified, and properly cited.

Received: 2013.12.24; Accepted: 2014.03.I4; Published: 2014.04.16

\begin{abstract}
BACKGROUND: Cervical cancer is one of the most common cancers in women worldwide, often associated with the infection of human papillomavirus (HPV). Toll-like receptor 8 (TLR8), a pattern recognition receptor, is involved in viral nucleic acid sensing. Recently TLR8 has been shown to be expressed in cancer cells, and it has been suggested that it may help cancer cell growth and tumor development. The objective of this study is to investigate the expression of TLR8 expression and its relationship with $\mathrm{Bcl}-2$ and VEGF in cervical cancer cells.

METHODOLOGYIPRINCIPAL: The mRNA expression levels of Bcl-2, VEGF and TLR-7,-8,-9 in newly diagnosed cervical cancer patients were detected by quantitative real-time PCR (qRTPCR). Epifluorescence microscope was used to determine the presence of TLR8 protein in Hela cells. The cell cycle and apoptosis were analyzed by flow cytometer, and the cell proliferation was measured by MTT assay. Our data showed the increased mRNA levels of TLR8 in human cervical cancer samples as well as in HeLa cells, a cell line derived from a human cervical cancer. In addition, there was a positive correlation between the expression levels of TLR8 and Bcl-2 and VEGF in cervical cancer patients. When Hela cells were treated with TLR8 agonist CL075, the percentage of cells in G2/M +S was remarkably increased, accompanied by increased COX-2, BCL-2 and VEGF mRNA levels.

CONCLUSIONS/SIGNIFICANCE: The mRNA expression level of TLR8 in the patients with cervical cancer and Hela cells were up-regulated, it consistent with the increased expression of VEGF and $\mathrm{Bcl}-2$. The results suggest that TLR8 may be an interesting therapeutic target in cervical cancer.
\end{abstract}

Key words: TLR8; Bcl-2; VEGF; human cervical cancer

\section{Introduction}

Cervical cancer is the third most common cancer in women worldwide, with $85 \%$ of cases occurring in developing countries. In China, it is estimated that 131,500 new cervical cancer cases occur annually, representing approximately one fifth of cases worldwide. Human papillomavirus (HPV) infection plays a crucial role in the development of cervical lesions and tumors [1-4]. HPV is detected in almost $100 \%$ of women with invasive cervical cancer; and infection with a high-risk HPV, such as the most prevalent HPV 16 and 18 subtypes, accounts for $76.7 \%$ of cervical cancer cases in China. DNAs from these HPV subtypes are frequently integrated into the host cell genomes in noninvasive squamous intraepithelial le- 
sions and squamous cell carcinomas. This integration is believed to be crucial in the induced expression of pattern recognition receptors (PRRs) for pathogen [5].

Toll-like receptors (TLRs) are pattern recognition receptors that recognize conserved pathogen-associated molecular patterns (PAMPs). Thirteen genes of the TLR family have been identified in humans and mice. These genes encode proteins which can recognize structurally conserved molecules derived from different microorganisms and activate immune cell responses [6-7]. TLR7 and TLR8, which detect viral single stranded (ss) RNA, and TLR9, which detects double stranded (ds) DNA, are located in the endosome [1-2]. TLRs are present in dendritic cells, macrophages, natural killer cells and other immune cells, and they have been identified as key components of the pathogen recognition process in human inflammatory responses including innate and adaptive immunity. In recent years, it has been found that TLRs are not only expressed in immune cells, but also in tumor cells [8]. The role of TLRs expression in tumor cells and its relationship with tumor development become a new research focus in tumor immunity. For example, Cherfils-Vicin et al. showed that TLR7 and TLR8 were expressed in human lung cancer cells, and loxoribine (TLR7 ligand) or PolyU (TLR8 ligand) can up-regulate B-cells lymphoma-2 (Bcl-2) and promote the survival of lung cancer cells [9].

Proteins of the Bcl-2 family regulate the apoptosis pathway and autophagy. The dysregulation of apoptosis is a key step in developing cancers, and mediates resistance to cancer therapy. Dysregulation of Bcl-2 family has been demonstrated in a wide spectrum of human cancers. Therefore, targeting the Bcl-2 family of proteins represents a promising therapeutic approach for malignancies. VEGF is the most potent angiogenic factor in tumour angiogenesis. A lot of studies have suggested that vascular endothelial growth factor (VEGF) expression in tumour tissue is significantly correlated with microvessel density and poor prognosis in human cancers including cervical cancer. Our current study aims to determine the level of TLR8 expression in the patients with cervical cancer and Hela cells, and to examine the relationship between TLR8 and Bcl-2 or VEGF expression in the development of cervical cancer.

\section{Materials and Methods}

\section{Patients and healthy controls}

The cancer tissues from fifteen newly diagnosed cervical cancer patients were used in the current study. These patients ranged from 36 to 75 years of age (average age, 47 years). Ten cervical tissues from cervical biopsy patients without cancer nor endome- triosis, age ranged from 34 to 65 years (average age, 46 years) were used as the controls. No subject was treated preoperatively or had a history of autoimmune disease, and no control had a prior history of cancer. This study was approved by the research ethics committee of the Affiliated Hospital of Jiangsu University, and written informed consent was obtained from all individuals.

\section{Reverse transcription-PCR (RT-PCR) and quantitative real-time PCR (qRT- PCR)}

RNA samples were extracted from cancer or control specimen using TRIzol (Invitrogen Life Technologies, California, USA), total RNA was isolated and reversed-transcribed with ReverTra Ace qPCR-RT kit (TOYOBO, Osaka, Japan) according to the manufacturers' instructions. RT-PCR and qRTPCR were performed as described previously [10]. The sequences for the primers used were: $\beta$-actin, 5'-CACGAAACTACCTTCAACTCC-3' (forward), 5'-CATACTCCTGCTTGCTGATC -3' (reverse); TLR7, 5'-CCTTTCCCAGAGCATACAGC-3' (forward), 5'-GGACAGAACTCCCACAGAGC-3' (reverse); TLR9, 5'-GGACCTCTGGTACTGCTTCCA-3' (forward), 5'-AAGCTCGTTGTACACCCAGTCT-3' (reverse); TLR8, 5'-CAGAGCATCAACCAAAGCAA-3' (forward), 5'-GCTGCCGTAGCCTCAAATAC-3' (reverse); COX-2, 5'-CGAGGTGTATGTATGAGTGTGG GAT-3' (forward), 5'-CCTTGAAGTGGGTAAGTATG TAGTG-3' (reverse); Bcl-2, 5' -TCCATGTCTTTGGAC AACCA-3' (forward), 5'-CTCCACCAGTGTTCCCAT CT-3' (reverse); VEGF, 5' -AGGAGGGCAGAATCAT CACG-3' (forward), 5' -CAAGGCCCACAGGGATTT TCT-3' (reverse). Relative quantification of mRNA expression was calculated by the comparative threshold cycle $(\mathrm{Ct})$ method.

\section{Cell culture and flow cytometry}

Hela cell, a kind of human cervical carcinoma cell line preserved in our laboratory, was cultured in DMEM medium, supplemented with $100 \mathrm{U} / \mathrm{ml}$ penicillin, $100 \mathrm{mg} / \mathrm{L}$ streptomycin and 10\% FBS (GIBCO, California, USA). For analyzing the effect of TLR8 agonist on cell cycle, $1 \times 10^{6}$ cell suspensions were stimulated by CL075 (a thiazoloquinoline compound, used as TLR8 agonist) in different concentrations as indicated. After $24 \mathrm{~h}$ treatment, the cells were collected by centrifugation at $4^{\circ} \mathrm{C}, 1000 \mathrm{r} / \mathrm{min}$ for $10 \mathrm{~min}$. The cells were washed twice with PBS, and fixed with $70 \%$ cold ethanol at $4^{\circ}$ Covernight followed by digestion with $0.2 \mathrm{mg} / \mathrm{ml}$ of RNaseA for $30 \mathrm{~min}$. Flow cytometry was performed using FACSCalibur Flow Cytometer (Becton Dickinson, Sparks, MD, USA) following PI staining. Apoptosis was analyzed under the similar conditions. In briefly, $1 \times 10^{5}$ cells were incubated with 
CL075 at $37^{\circ} \mathrm{C}$ for $48 \mathrm{~h}$, and stained by PI for flow cytometry analysis.

\section{Immunofluorescence}

$5 \times 10^{4}$ cells inoculated on coverslip were washed twice with PBS and fixed with $4 \%$ paraformaldehyde at $37^{\circ} \mathrm{C}$ for $1 \mathrm{~h}$. Triton-X100 was added for rupture membrane at room temperature for $30 \mathrm{~min}$. The coverslip was then immersed in blocking buffer with 3\% BSA for $60 \mathrm{~min}$. TLR8 antibody (Abcam Company, Britain) was applied for $2 \mathrm{~h}$ at room temperature. After washing, PE-labeled goat anti- mouse IgG (MultiSciencesBiotechCo., Ltd, China) was added at $37^{\circ} \mathrm{C}$ for 45 min, followed Hochest 33258 staining at $37^{\circ} \mathrm{C}$ for 15 min. The cells were examined with a fluorescence microscope (Olympus BX51, Tokyo, Japan) and analyzed using the Image J software.

\section{Cell Proliferation Assay}

Cells were seeded into 96 -well plates at $1 \times 10^{5}$ cells $/ \mathrm{ml}\left(2 \times 10^{4}\right.$ cells per well $)$ and cultured for $3 \mathrm{~h}$ at $37^{\circ} \mathrm{C}$ in an air $5 \% \mathrm{CO}_{2}$ atmosphere. After the cells attached to the plate, CL075 in final concentration of $0.1 \mu \mathrm{g} / \mathrm{ml}, 0.5 \mu \mathrm{g} / \mathrm{ml}, 1.0 \mu \mathrm{g} / \mathrm{ml}$ or $2.5 \mu \mathrm{g} / \mathrm{ml}$ was respectively added to the wells; the cells were incubated at $37^{\circ} \mathrm{C}$ for $24 \mathrm{~h}, 48 \mathrm{~h}$, or $72 \mathrm{~h}$, respectively. At each time point, $20 \mu \mathrm{L}(5 \mathrm{mg} / \mathrm{mL})$ of MTT (Sigma, USA) was added to each well, and the plate was further incubated for $4 \mathrm{~h}$ to deoxidize MTT under light-blocking condition. After removal of the MTT dye solution, cells were treated with $150 \mu \mathrm{L}$ DMSO, and the absorbance at $490 \mathrm{~nm}$ was measured using ELX $800 \mathrm{UV}$ reader (BioTek, Winooski, VT, USA). This cell proliferation assay was performed in triplicate, and repeated at least twice.

\section{Statistical analysis}

Data were expressed as the mean $\pm S D$. Statistical analysis was performed using Prism5 (GraphPad Software). All experiments were performed at least in twice. Statistical differences between groups were compared by one-way analysis of variance followed by Dunnett's multiple comparison tests to identify significantly different results. Pearson method was used for correlation analysis. $\mathrm{P}$ values less than 0.05 were considered significant.

\section{Results}

\section{Expression of TLR8 in human cancer cell lines}

Thirteen human cancer cell lines, including 95D, HepG2, NICH446, U266, SGC, HCT-8, BGC, SW620, A549, HT1080, SKOV-3, U937 and Hela were examined for TLR8 expression. Our data showed that SKOV-3, U937 and Hela expressed TLR8 mRNA at high levels. Low TLR8 levels were found in SGC, HCT-8, BGC, SW620, A549 and HT1080; but no TLR8 mRNA was detected in 95D, HepG2, NICH446 or U266 (Figure 1). The expression level of TLR8 mRNA in Hela cell line was higher than that in the other cell lines (Figure 1). Consistently, immunostaining showed presence of TLR8 protein in Hela cells (Figure 2A).
$\Delta$

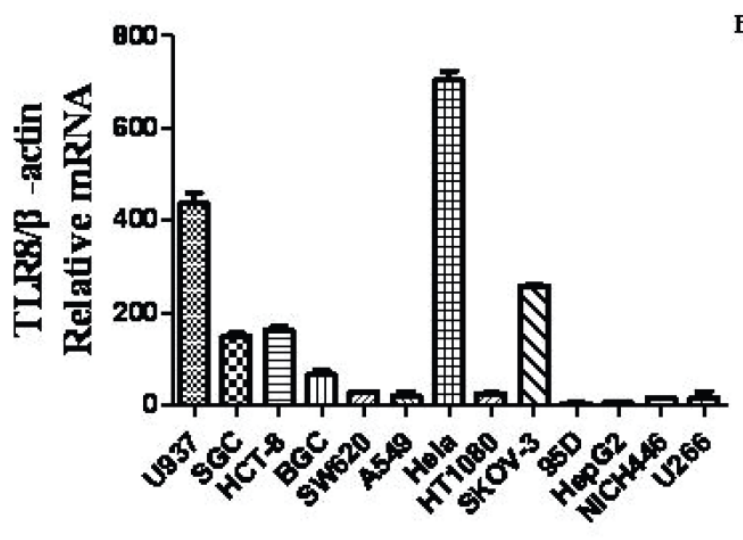

B

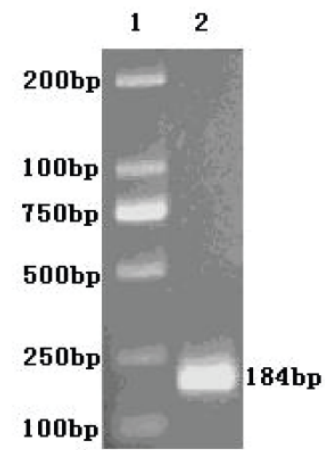

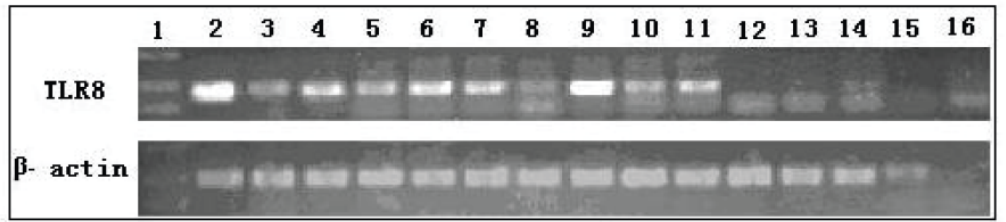

Fig.I The expression of TLR8 in various human cancer cell lines. The levels of TLR8 mRNA in 13 human cancer cell lines were detected by qRT-PCR. A: Relative TLR8 mRNA levels in each cell line as determined by qRT-PCR. B: RT-PCR product of TLR8 from Hela cells on agarose gel. Lane I, DNA marker DL2000, Line 2, the PCR product of TLR8. C: RT-PCR products of TLR8 in 13 human cancer cell lines. Line I, DNA marker DL2000; Line 2, positive control; Line 3 to Line I5, U937, SGC, HCT-8, BGC, SW620, A549, Hela, HTI080, SKOV-3, 95D, HepG2, NICH446 and U266; Line 16, negative control. 
A

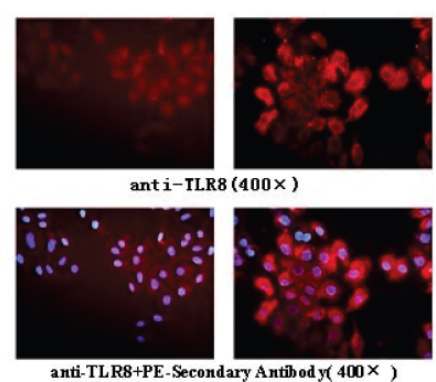

$\mathrm{C}$

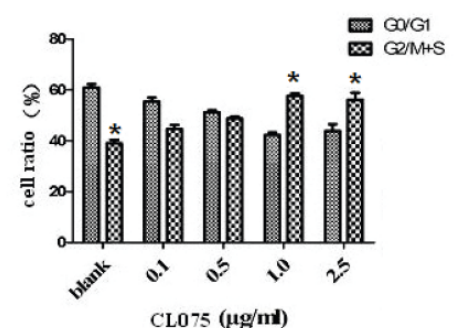

B

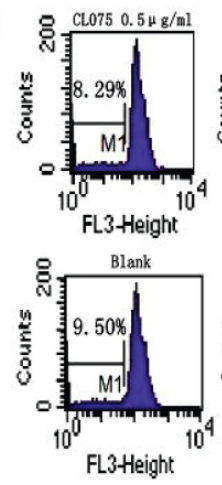

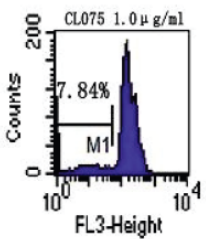
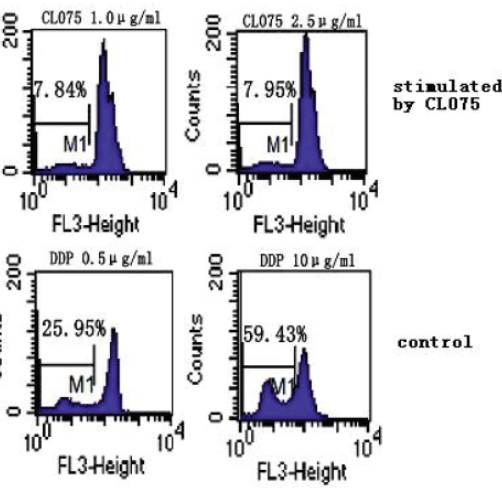

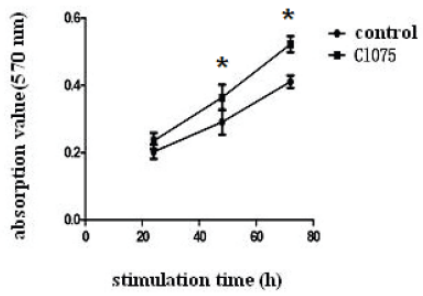

Fig.2 Intracellular localization of TLR8 and the effect of CL075 stimulation in Hela cells. A: Immunostaining showed presence of TLR8 protein in Hela cells. It is suggestive that TLR8 protein exists in the cytoplasm of Hela cells. B: The cell cycle profiles of Hela cells after CL075 treatment determined by flow cytometry. The top three panel shows the cell cycle profiles of cells treated with different concentration of CL075 as indicated; the lower panels shows the effect of blank control or DDP treatment. C: The changes in cell cycle population after CL075 treatment of Hela cells. D: The time-course effects of CL075 on the cell proliferation in Hela cells. *P<0.05.

\section{The effect of TLR8 agonist CL075 on Hela cells}

CL075, a thiazoloquinoline compound, used as TLR8 agonist. Treatment of HeLa cells with CL075 for $48 \mathrm{~h}$ resulted increases in the percentage of $\mathrm{G} 2 / \mathrm{M}+\mathrm{S}$ phase, suggesting an increase in cell proliferation (Figure 2B and 2C). This was confirmed by MTT assay (Figure 2D). Unlike DDP, CL075 did not induce apoptosis in Hela cells (Fig.2B). The levels of COX-2, BCL-2, and VEGF mRNA was significantly increased after CL075 treatment for $24 \mathrm{~h}$, and reached to peak at $48 \mathrm{~h}$ in Hela cells (Figure 3).

\section{Enhanced expression levels of TLR7 and TLR8 in cancer tissues from patients with cervical carcinoma}

Quantitative RT-PCR showed higher mRNA levels of TLR7 and TLR8 in cervical cancer samples from patients than that in cervical tissues from patients without cancer. In contrast, there was no clear difference in TLR9 expression in tissue samples from the cancer patients and controls (Figure 4). Bcl-2 and VEGF expression levels were significantly increased in cancer tissues from the patients with cervical cancer (Figure 4).

\section{Correlation analysis between TLR8 and $\mathrm{Bcl}-2$ or VEGF expression in cancer tissues}

Using the method of Spearman analysis, we found that there was a positive correlation between the expression level of TLR8 and Bcl-2 or VEGF in cervical cancer samples. No correlation was found between the TLR7 and Bcl-2 or VEGF mRNA (Figure 5).

\section{Discussion}

TLRs play an important role both in innate and adaptive immune response, they express in dendritic cells, macrophages, natural killer and other immune cells to recognize pathogenic microorganisms and damaged cells, and contribute to anti-infective or anti-tumor effects [11-12]. However, recent studies revealed that tumor cells can also express TLRs. TLRs-expressed tumor tissues and cells not only hinder the infiltration of immune cells, but also can change the type of inflammation, therefore facilitate tumor occurrence and development [13]. The role of TLRs expressed in tumor cells has become a new research focus in tumor immunity [14-16]. The potential for developing new cancer treatments is stated that TLR7 or TLR8 agonist, is approved for treatment of genital warts and has been suggested for treatment of other HPV-associated disease including papillomavirus infection [1, 17-18]. Recent studies show that TLR7 and TLR8 are expressed in human lung cancer cells, TLR7 ligand (loxoribine) and TLR8 ligand (PolyU) are able to up-regulate $\mathrm{Bcl}-2$ expression and to promote the survival of lung cancer cells [2]. In this study, we also found increased expressions of TLR7 and TLR8 in cervical cancer cells, suggesting that the TLRs mediated tumor immunity function was compromised in these cancer patients. 

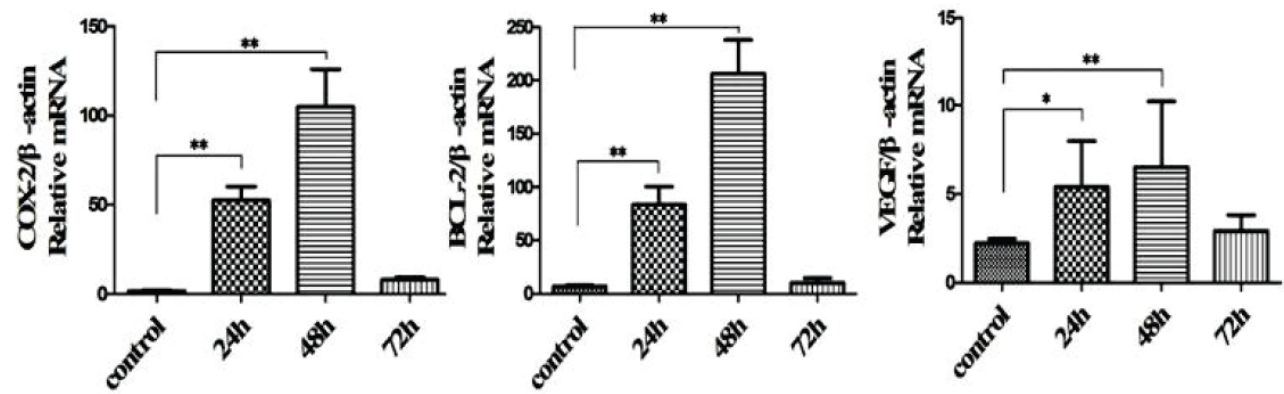

Fig.3. Expression of COX-2, Bcl-2 and VEGF in CL075-treated Hela cells. The mRNA levels of COX-2, Bcl-2 and VEGF in CL075-stimulated human cervical carcinoma cell line were detected by $q R T-P C R$. These data were expressed as means \pm SD of three independent experiments. $* P<0.05, * * P<0.01$.
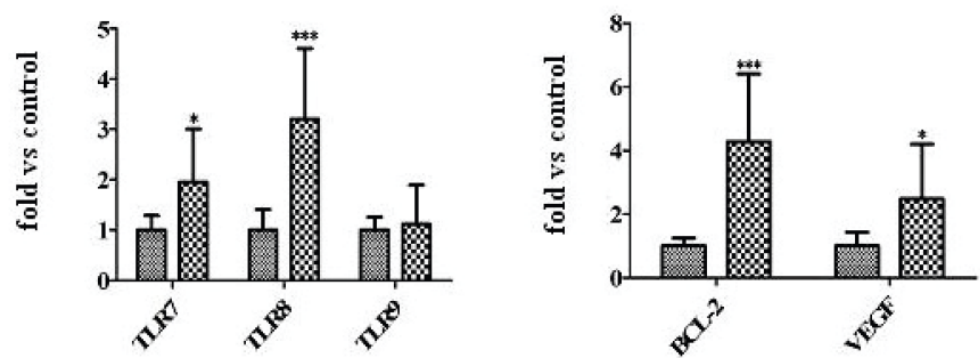

healthy control

$\mathrm{B}$ cervical cancer

Fig. 4. The expression of TLR7/8/9, Bcl-2, and VEGF mRNA in cervical cancer tissues. The mRNA levels of TLR7/TLR8/TLR9 and Bcl-2/VEGF in cervical cancer tissues from patients were analyzed by $\mathrm{qRT}$-PCR. The data are expressed as means \pm SD of three independent experiments. *P<0.05, ***P $<0.001$.

A

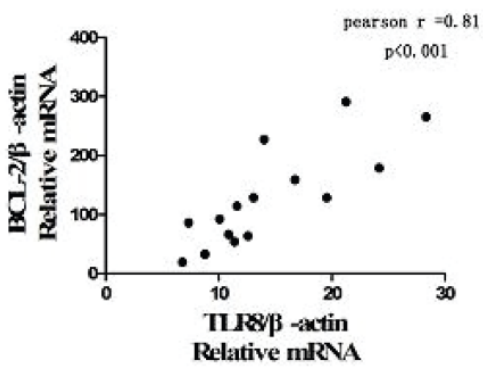

$\mathrm{C}$

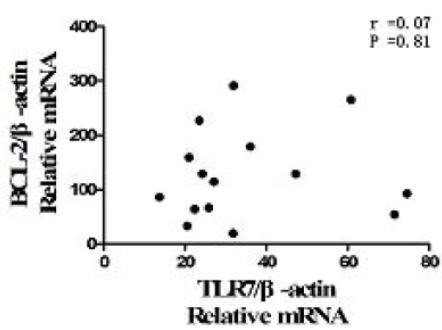

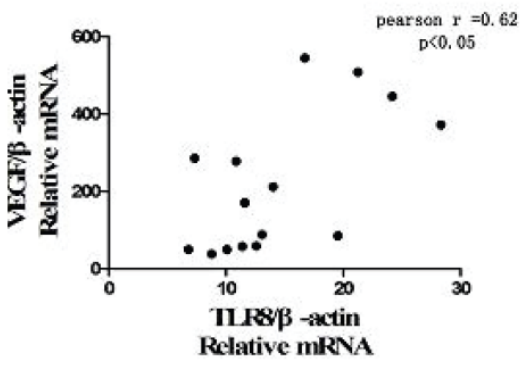

D

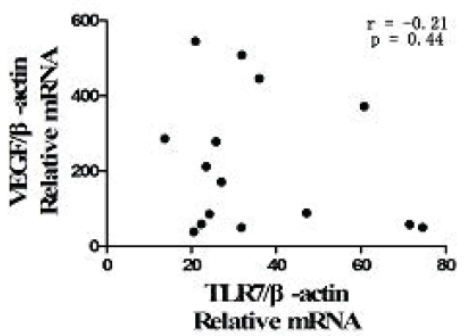

Fig. 5 The correlation between TLR8 and Bcl-2/VEGF mRNA level in cervical cancer tissues from patients. The correlation between TLR7/TLR8 and Bcl-2/VEGF mRNA level in cervical cancer tissue was analyzed with Pearson method. A: The positive correlation was found between TLR8 and Bcl-2 expression $(r=0.81, P<0.001) ; B$ : The correlation between TLR8 and VEGF expression $(r=0.62, P<0.05)$; : The correlation between TLR7 and Bcl-2 $(r=0.07, P=0.81)$; $D$ : The correlation between TLR7 and VEGF $(r=-0.21, P=0.44)$.

The Bcl-2 gene product is thought to contribute to oncogenesis by suppressing signals that induce apoptotic cell death, and a lot of studies have suggested that VEGF expression is significantly correlated with poor prognosis in human cancers [19]. In the present study, these two genes were selected, aims to analyze the expression of TLR8 in human cervical cancer biopsies and a possible correlation with tumor associated genes and evaluate the possible development trends of cervical cancer. We observed a positive correlation between TLR8 expression and Bcl-2 or VEGF expression in cervical cancer tissues and Hela cells. VEGF and Bcl-2 have been shown to play important roles in angiogenesis, a critical feature for 
tumor development. A recent report found that Bcl-2, as an inhibitor of apoptosis, had prognostic value in classical Hodgkin lymphoma [20]. The predominant regulator of tumor angiogenesis is VEGF, which has become an important target in the development of novel anti-tumor strategies [21-22], although the clinical effectiveness of these new strategies remains to be seen [23]. The increased VEGF and Bcl-2 expression reported here suggests that future therapeutic approaches targeting on angiogenesis can be used in cervical cancer patients.

Similar to human primary cervical cancer samples, the human cervical carcinoma cell line Hela also expressed high levels of TLR8, Bcl-2, and VEGF, therefore could be used as an excellent model to investigate the functions and mechanisms of TLR in cervical cancer cells. Our data showed that the percentage of cell in G2/M+S had a remarkably enhancement in Hela cells after treatment of CL075, a TLR8 ligand, indicating that the binding of TLR8 to its ligand stimulated cell proliferation. Furthermore, an increased expression of COX-2, BCL-2 and VEGF mRNA was observed in CL075 treated Hela cells, suggesting the activation of angiogenesis and inhibition of apoptosis, all of which may contribute to tumor development.

In conclusion, our data offer the convincing evidence for first time that the TLR8 mRNA was upregulated both in cervical cancer tissues and Hela cells, it consistent with the increased expression of VEGF and Bcl-2 which correlated with poor prognosis in human cancers. Our study reveal that the high TLR8-expressed cervical cancer cells may strongly correlate with carcinogenesis and tumor invasion by inhibiting TLR8 positive immune cells to recognize tumor or viral antigen, which influenced anti-tumor immune response. Further investigations of its roles and mechanisms will provide critical information not only to help understand cervical cancer pathogenesis, but also useful for the future development of novel targeted therapeutic strategies. For example, it will be important to investigate whether TLR8 antagonists can affect cervical cancer cell proliferation and in vivo tumor growth. Our further studies will focus on the exploration of TLR8 in intraepithelial lymphocytes and tumor macrophages using stratifying samples with different cervical cancer grades. In short, understanding the functions of TLRs in cancer patient will be important for the developing new cancer immunotherapies.

\section{Acknowledgments}

The authors thank Dr Caixia Sun and Yingzhao Liu for technical assistance. This work was supported by grants from the National Natural Science Founda- tion of China (31270947, 31170849 and 30972748), Natural Science Foundation of Jiangsu Province (BK2011472) and Postdoctoral Foundation of China (2012M511705) and The Peak of six personnel in Jiangsu (2013-WSN-002).

\section{Competing Interests}

The authors have declared that no competing interest exists.

\section{References}

1. Daud II, Scott ME, Ma Y, et al. Association between toll-like receptor expression and human papillomavirus type 16 persistence. Int J Cancer. 2011; 128:879-86.

2. Heil $\mathrm{F}, \mathrm{Hemmi} \mathrm{H}$, Hochrein $\mathrm{H}$, et al. Species-specific recognition of single-stranded RNA via Toll-like receptor 7 and 8. Science. 2004; 303:1526-9.

3. Wang L, Dai SZ, Chu HJ, et al. Integration sites and genotype distributions of human papillomavirus in cervical intraepithelial neoplasia. Asian Pac J Cancer Prev. 2013; 14:3837-41.

4. Shen $\mathrm{XH}$, Liu SH. Human papillomavirus genotypes associated with mucopurulent cervicitis and cervical cancer in hangzhou, china. Asian Pac J Cancer Prev. 2013; 14:3603-6.

5. Zheng B, Morgan ME, van de Kant HJ, et al. Transcriptional modulation of pattern recognition receptors in acute colitis in mice. Biochim Biophys Acta. 2013 Dec;1832(12):2162-72.

6. Mantovani A, Allavena P, Sica A, et al. Cancer-related inflammation. Nature. 2008; 454:436-44.

7. Gribar SC, Richardson WM, Sodhi CP, et al. No longer an innocent bystander: epithelial toll-like receptor signaling in the development of mucosal inflammation. Mol Med. 2008; 14:645-59.

8. Sato $\mathrm{Y}$, Goto $\mathrm{Y}$, Narita N, et al. Cancer Cells Expressing Toll-like Receptors and the Tumor Microenvironment. Cancer Microenviron. 2009; 2: 205-14.

9. Cherfils-Vicini J, Platonova S, Gillard M, et al. Triggering of TLR7 and TLR8 expressed by human lung cancer cells induces cell survival and chemoresistance. J Clin Invest. 2010; 120:1285-97.

10. Xue $Y$, Yang Y, Su Z, et al. Local delivery of T-bet shRNA reduces inflammation in collagen II-induced arthritis via downregulation of IFN- $\gamma$ and IL-17. Mol Med Rep. 2014; 9:899-903.

11. Reynolds JM, Dong C. Toll-like receptor regulation of effector T lymphocyte function. Trends Immunol. 2013; S1471-4906(13)00093-8.

12. Liang J, Fu J, Kang H, et al. The stimulatory effect of TLRs ligands on maturation of chicken bone marrow-derived dendritic cells. Vet Immunol Immunopathol. 2013; S0165-2427(13)00196-7.

13. Renga B, Mencarelli A, Cipriani S, et al. The bile acid sensor FXR is required for immune-regulatory activities of TLR-9 in intestinal inflammation. PLoS One. 2013; 8:e54472.

14. Ma Y, Li J, Chiu I, et al. Toll-like receptor 8 functions as a negative regulator of neurite outgrowth and inducer of neuronal apoptosis. J Cell Biol. 2006; 175:209-15.

15. Gorden KK, Qin XX, Binsfeld CC, et al. Cutting edge: activation of murine TLR8 by a combination of imidazoquinoline immune response modifiers and polyT oligodeoxynucleotides. J Immunol. 2006; 177:6584-7.

16. Demaria O, Pagni PP, Traub S, et al. TLR8 deficiency leads to autoimmunity in mice. J Clin Invest. 2010; 120:3651-62.

17. Zhou $\mathrm{Q}$, Zhu $\mathrm{K}$, Cheng $\mathrm{H}$. Tool-Like receptors in Human Papillomavirus Infection. Arch Immunol Ther. 2013; 61:203-15.

18. Lu H, Dietsch GN, Matthews MA, et al. VTX-2337 Is a Novel TLR8 Agonist That Activates NK Cells and Augments ADCC. Clinical Cancer Res. 2011; 18:499-509.

19. Zhang M, Shan H, Chang P, et al. Hydrogen sulfide offers neuroprotection on traumatic brain injury in parallel with reduced apoptosis and autophagy in mice. PLoS One. 2014; 9:e87241.

20. Koh YW, Park C, Yoon DH, et al. Prognostic Significance of COX-2 Expression and Correlation With Bcl-2 and VEGF Expression, Microvessel Density, and Clinical Variables in Classical Hodgkin Lymphoma. Am J Surg Pathol. 2013; 37:1242-51.

21. Kumar MM, Adurthi S, Ramachandran S, et al. Toll-like receptors 7, 8, and 9 expression and function in primary human cervical cancer Langerhans cells: evidence of anergy. Int J Gynecol Cancer. 2013; 23:184-92.

22. Riddell JR, Maier P, Sass SN, et al. Peroxiredoxin 1 stimulates endothelial cell expression of VEGF via TLR4 dependent activation of HIF-1a. PLoS One. 2012; 7:e50394.

23. Zhou S, Yang Y, Yang Y, et al. Combination Therapy of VEGF-Trap and Gemcitabine Results in Improved Anti-Tumor Efficacy in a Mouse Lung Cancer Model. PLoS One. 2013; 8: e68589. 